\title{
Competing endogenous RNA network analysis reveals pivotal ceRNAs in bladder urothelial carcinoma
}

\author{
Yangle Li, Xiongbing Zu, Xiheng Hu, Cheng Zhao, Miao Mo, Benyi Fan \\ Department of Urology, Xiangya Hospital, Central South University, Changsha, China \\ Contributions: (I) Conception and design: All authors; (II) Administrative support: B Fan; (III) Provision of study materials or patients: None; (IV) \\ Collection and assembly of data: Y Li, X Zu, X Hu; (V) Data analysis and interpretation: Y Li, X Zu, C Zhao, M Mo; (VI) Manuscript writing: All \\ authors; (VII) Final approval of manuscript: All authors. \\ Correspondence to: Benyi Fan. 87 Xiangya Road, Changsha 410008, China. Email: fby2003@csu.edu.cn.
}

\begin{abstract}
Background: Bladder urothelial cancer (BUC) has become one of the most frequently occurring malignant tumors worldwide and it is of great importance to explore the molecular pathogenesis of bladder cancer. Emerging evidence has demonstrated that dysregulation of noncoding RNAs is critically involved in the tumorigenesis and progression of BUC. Long noncoding RNAs (lncRNAs) can act as microRNA (miRNA) sponges to regulate protein-coding gene expression and therefore form a competing endogenous RNA (ceRNA) network. ceRNA networks have been proven to play vital roles during tumorigenesis and progression. Elements involved in the ceRNA network have also been identified as potential therapeutic targets and prognostic biomarkers in various tumors. Understanding the regulatory mechanisms and functional roles of the ceRNA system will help understand tumorigenesis, progression mechanisms of BUC and develop therapeutics against cancer.
\end{abstract}

Methods: In this study, we utilized the TCGA database and analyzed the multilevel expression profile of BUC. ceRNA regulatory networks were constructed by integrating tumor progression and prognosis information. RNA immunoprecipitation (RIP) and qRT-PCR were applied to verify the identified ceRNA networks. KEGG enrichment analysis was implemented to infer the biological functions of the regulatory system.

Results: We identified a lncRNA-miRNA-mRNA regulatory ceRNA network containing two lncRNAs, one miRNA and 14 mRNAs. The ceRNA network we identified showed significant roles in BUC tumorigenesis, progression, and metastases.

Conclusions: The proposed ceRNA network may help explain the regulatory mechanism by which lncRNAs function as ceRNAs and improve our understanding of the pathogenesis of BUC. Moreover, the candidate elements involved in the ceRNA network can be further evaluated as potential therapeutic targets and prognostic biomarkers for BUC.

Keywords: Competing endogenous RNAs (ceRNAs); bladder urothelial cancer (BUC); progression; metastases; biomarkers

Submitted Aug 12, 2020. Accepted for publication Dec 13, 2020.

doi: $10.21037 /$ tau-20-1167

View this article at: http://dx.doi.org/10.21037/tau-20-1167

\section{Introduction}

Bladder urothelial cancer (BUC) is one of the most common cancers and occurs more frequently in men and older people. Men (35.2 new cases per 100,000 persons) are approximately three times more likely than women (8.7 new cases per 100,000 persons) to develop BUC. BUC is most frequently diagnosed among people aged 65 to 74 years old, with a median age of 73 . BUC is fairly common and ranks as the tenth leading cause of cancer death in the 
United States (1). BUC can be mainly classified into three types: urothelial carcinoma, squamous cell carcinoma, and adenocarcinoma. Among the types mentioned above, urothelial carcinoma (BUC) is the most common subtype, and its occurrence, as well as progression, has attracted the most attention of the research and clinical fields.

Noncoding RNAs are functional transcripts that function directly as structural, catalytic or regulatory RNA molecules but are not translated into proteins. Long noncoding RNAs (lncRNAs) are ncRNAs more than $200 \mathrm{bp}$ in length and play diverse roles in regulating gene expression at both the transcriptional and translational levels. LncRNAs have been reported to be involved in tumorigenesis and tumor progression. Previous studies have shown that lncRNAs regulate cellular processes in different ways, including interacting with proteins, mRNAs, miRNAs and DNAs. For example, lncRNAs can act as signals to guide or scaffold epigenetic factors such as DNA methyltransferase and histone modification enzymes at the chromatin level to regulate gene expression (2-4). Competing endogenous RNAs (ceRNAs) refer to lnc/circRNAs that competitively occupy the shared binding sequence of miRNAs, thus sequestering the target miRNAs and regulating the expression of downstream interacting genes. ceRNA networks formed by lncRNA/miRNA/mRNA interactions have been verified to broadly participate in cancer. For example, in colorectal cancer (CRC), the ceRNA axes PVT1/miR-30d-5p/RUNX2 and PVT1/miR-455/ RUNX2 are involved in tumorigenesis, cell proliferation, invasion, tumor stages and lymph node metastasis. In CRC, overexpressed PVT1 binds to miR-30d-5p or miR-455 directly, thus relieving its repression to the downstream target oncogene RUNX2 $(5,6)$. circFBLIM1 functions as a ceRNA to regulate FBLIM1 expression by sponging miR-346 to exert regulatory functions in hepatocellular carcinoma (HCC) (7). In BUC, some studies have shown that ceRNA regulation is also involved in tumor progression and proliferation. Researchers have stated that the PVT1/ miR-128/VEGFC (8) and MALAT1/miR-124/foxq1 (9) axes are correlated with clinical progression and poor prognosis. LncRNA OXCT1-AS1 showed the ability to promote bladder cancer cell aggressiveness via miR-4555p/JAK1 signaling (10). LncRNA XIST can interact with miR-124 to modulate BUC growth, invasion, and migration by targeting androgen receptor (AR) (11). circMTO1 can suppress BUC metastasis by sponging miR-221 and inhibiting epithelial-to-mesenchymal transition (12).
The ceRNA network may be one of the most promising therapeutic targets against cancer. Targeting nonessential elements in the ceRNA network may activate alternative signaling pathways of cancer cells to overcome the damage induced by therapy. This leads to an ineffective therapeutic response and increase the risk of resistance to chemoand/or radiotherapy (13). Therefore, understanding the topology and dynamics of cancer ceRNA regulatory networks can help to identify hub elements in tumorigenesis and progression. Therapies targeting key miRNAs in the ceRNA network can impact not only the feedback pathways of miRNAs but also compensatory pathways with common MREs (miRNA recognition elements).

Multi-omics sequencing projects have made the screening of the ceRNA network a reality. Here, in this study, we aimed to identify a ceRNA network composed of lncRNAs-miRNAs-mRNAs in BUC that function during tumorigenesis and are correlated with progression.

We present the following article in accordance with the STROBE reporting checklist (available at http://dx.doi. org/10.21037/tau-20-1167).

\section{Methods}

\section{Data sources}

The expression data for lncRNAs, mRNAs, and miRNAs in BUC tumors, as well as normal samples, were downloaded from UCSC Cancer Browser (https://xenabrowser.net/) under the GDC TCGA Bladder Cancer (BLCA) category. Pathological and survival time data were also downloaded from the same repository.

\section{Differential expression analysis}

A total of 430 bladder samples, 411 from tumor sites and 19 from normal sites, were subsequently used for differential expression analysis of mRNAs and lncRNAs. The $\mathrm{R}$ package limma (14) with its voom method, linear modeling and empirical Bayes moderation were applied to assess differentially expressed genes. miRNA data for 413 normal and 19 tumor BUC samples were downloaded from the same repository. The RPM data were first quantile normalized and subsequently subjected to a standard limma procedure for differential expression analysis. Differentially expressed lncRNAs, miRNAs and mRNAs were identified with adjusted $\mathrm{P}$ values $<0.05$ and $\mid \log \mathrm{FCl}>1$. 


\section{Prediction of lncRNA-miRNA and miRNA-mRNA interactions}

LncRNA-miRNA interaction predictions were performed using miRcode (http://www.mircode.org/) (15). Interactions between miRNA and mRNA were predicted using miRanda (http://www.microrna.org/) (16). To further improve the reliability of the interactions, expression correlation was also analyzed between each predicted pair, and a final pairwise association was chosen with a correlation coefficient $<-0.3$ and $\mathrm{P}$ value $<0.05$.

\section{Association of ceRNA network and patient prognosis}

Kaplan-Meier curves were used to assess the relationship between the expression of ceRNA network elements and BUC patient overall survival. Gene expression changes across tumor stages were analyzed using the GEPIA online tool (http://gepia.cancer-pku.cn/) (17).

\section{Cell lines}

The T24 and 5637 cell lines (human bladder cancer cell lines) were purchased from the American Type Culture Collection (ATCC; Manassas, VA). These cell lines cultured under a humidified atmosphere with $95 \%$ air $/ 5 \% \mathrm{CO} 2$ at $37^{\circ} \mathrm{C}$ in Dulbecco's modified essential medium (DMEM; Gibco, Grand Island, NY) with 10\% fetal bovine serum (FBS, Gibco), $1 \%$ streptomycin $(100 \mu \mathrm{g} / \mathrm{mL})$ and penicillin $\mathrm{G}(100 \mathrm{U} / \mathrm{mL})$. The medium was changed every three day.

\section{RNA immunoprecipitation (RIP)}

The RIP assay was used to explore the relationships among lncRNA-miRNA-mRNA. The EZ-Magna RIP RNA Binding Protein Immunoprecipitation Kit (Millipore) was used for this assay. Briefly, Cultured T24 and 5637 cells were lysed in RIP lysis buffer to isolate the nuclei. The chromatin was sheared by homogenization and then mixed with magnetic beads which had bind the antibodies against human Ago2 or control IgG (Millipore). After RNA purification, the products were analyzed by qRT-PCR.

\section{Quantitative real-time polymerase chain reaction (qRT-PCR)}

The complementary DNA (cDNA) synthesis through the reverse transcription kit (Takara, Kyoto, Japan) following the instruction. For the quantitative analysis, this assay was performed using SYBR Green PCR Master Mix (Takara) on the Step-One Plus Real-Time PCR System (Applied Biosystems). The quantitative PCR was performed under the following conditions: initial denaturation at $95^{\circ} \mathrm{C}$ for $5 \mathrm{~min}$, 40 cycles of denaturation at $95^{\circ} \mathrm{C}$ for $15 \mathrm{~s}$, annealing at $60{ }^{\circ} \mathrm{C}$ for $1 \mathrm{~min}$, and final extension at $72{ }^{\circ} \mathrm{C}$ for $30 \mathrm{~s}$. The specific primer set were listed as below: Forward Primer for NR2F1-AS1, 5'-TGGAGAGGTGAAACAGTCGC-3', Reverse Primer for NR2F1-AS1, 5'-GACCAGGGGACAC CATTGAG -3'; Forward Primer for AC000403, 5'-GCAA GGgatTgGgGagGaTT-3', Reverse Primer for AC000403, 5'-ACAGGTTACTTGCAGGCACA-3'; Forward Primer for RP11-456K23, 5'-TTTGGCCTACA AAGGGGGTC-3', Reverse Primer for RP11-456K23, 5'-TTGCAGACCTTCCCGAATCC-3'. Primers for hsalet-7 $\mathrm{g}$ was bought from QIAGEN.

\section{Functional analysis}

The pathways of genes involved provide vital functional information of the ceRNA network. Thus, we performed Kyoto Encyclopedia of Genes and Genomes (KEGG) pathway enrichment analysis using the $\mathrm{R}$ package clusterProfiler (18) to identify functional attributes. Adjusted $\mathrm{P}<0.1$ was set as the cut-off criterion.

\section{Statistical analysis}

All statistical analysis was performed under $\mathrm{R}$ version 3.5.3. $\mathrm{P}$ values $<0.05$ and $\mid \log \mathrm{FCl}>1$ were considered as statistically significant for differential expression analysis between urothelial bladder carcinoma and adjacent tissues. Potential interactions between lncRNA-miRNA and mRNA-miRNA were selected using correlation coefficient $<-0.3$ and $\mathrm{P}$ value $<0.05$. Genes with prognostic value were selected using $\mathrm{P}$ value $<0.05$ with Kaplan-Meier survival analysis. Adjusted $\mathrm{P}$ value $<0.1$ was used to identify affected KEGG pathways of identified ceRNA network.

\section{Ethical statement}

The study was conducted in accordance with the Declaration of Helsinki (as revised in 2013). All data from TCGA are publicly available and their use do not require the approval of a local ethics committee. 

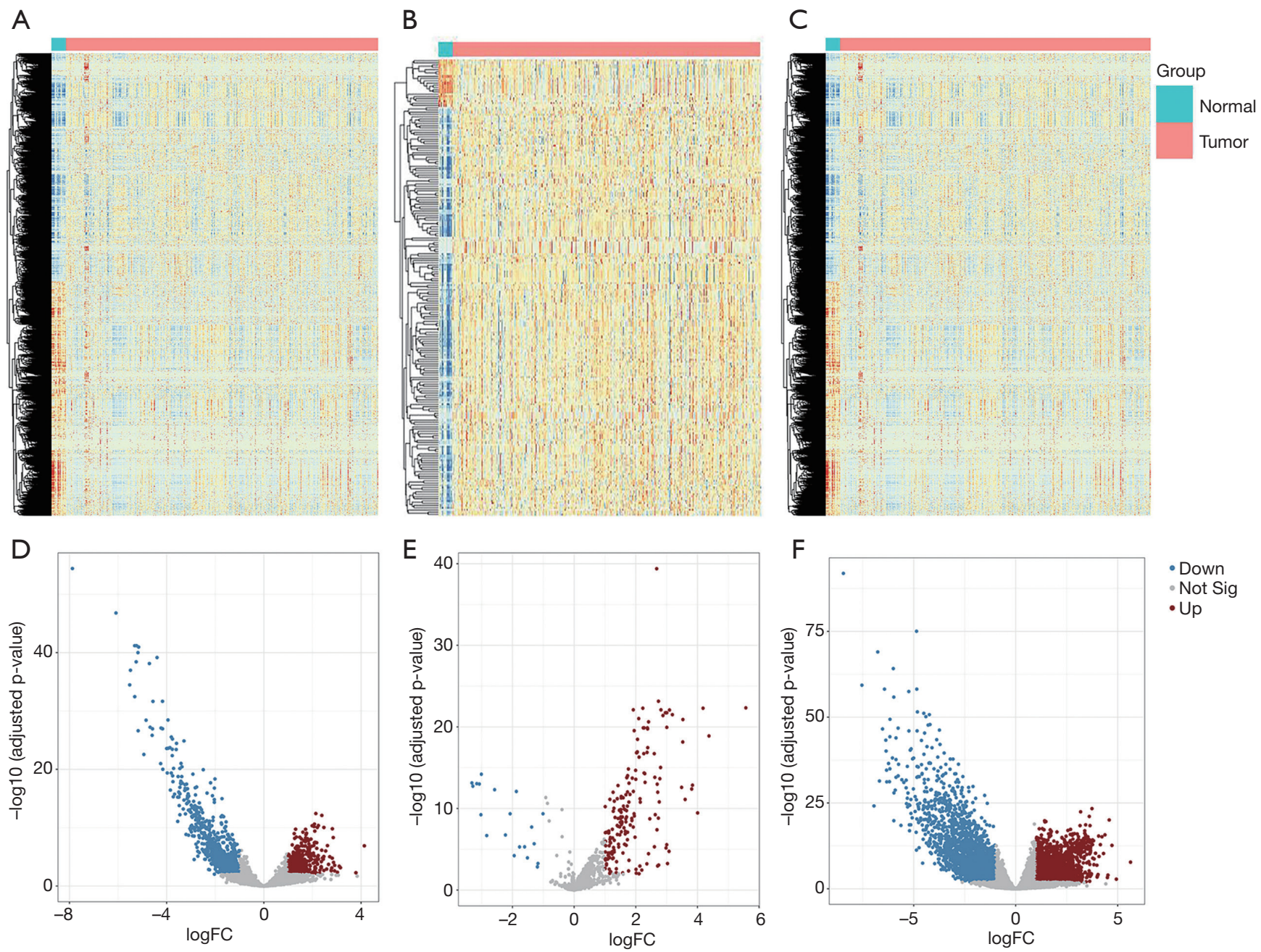

Figure 1 Differential expression analysis of lncRNAs, miRNAs and mRNAs. (A) Heatmap of 1,380 differentially expressed lncRNAs. (B) Heatmap of 203 differentially expressed miRNAs. (C) Heatmap of 4,909 differentially expressed mRNAs. (D) Volcano plot of lncRNAs. (E) Volcano plot of miRNAs. (F) Volcano plot of mRNAs. lncRNA, long noncoding RNA; miRNA, microRNA.

\section{Results}

\section{Differential expression analysis (Figure 1)}

The expression profile of mRNA, miRNA, and lncRNA between BUC tumor samples and adjacent normal samples were obtained from TCGA. Differentially expressed lncRNAs, miRNAs and mRNAs were selected using adjusted $\mathrm{P}$ values $<0.05$ and $|\log \mathrm{FC}|>1$ as cutoffs. Finally, a total of 1,380 differentially expressed lncRNAs (DElncRNAs, Figure 1A,D), 203 differentially expressed miRNAs (DEmiRNAs, Figure 1B,E) and 4,909 differentially expressed mRNAs (DEmRNAs, Figure 1C,F) were identified using the above criteria. Among them, the 1,380 differentially expressed lncRNAs included 461 upregulated lncRNAs and 919 downregulated lncRNAs in tumors compared with normal samples. The 203 differentially expressed miRNAs consisted of 182 upregulated miRNAs and 21 downregulated miRNAs. In addition, there were 2,390 upregulated and 2,519 downregulated mRNAs among the 4,909 differentially expressed mRNAs.

\section{Identification of DElncRNA-DEmiRNA and DEmiRNA- DEmRNA interactions}

Based on miRcode prediction and expression correlation, we selected DElncRNA-DEmiRNA pairs and successfully 


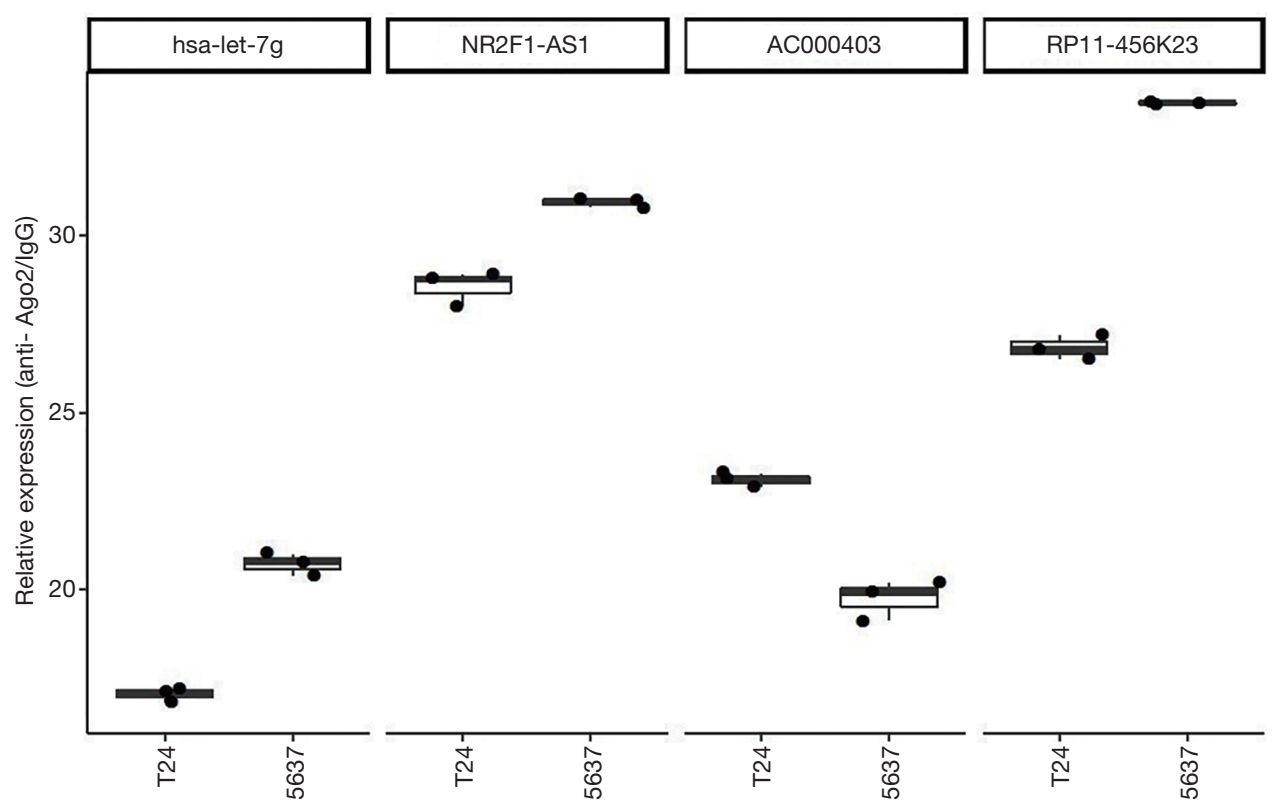

Figure 2 RIP assays confirmed the physical interactions between hsa-let-7g and NR2F1-AS1, AC000403, and RP11-456K23. qRT-PCR results showed significantly enriched hsa-let-7g, NR2F1-AS1, AC000403 and RP11-456K23 in the anti-Ago2 group compared to the IgG control group. qRT-PCR, quantitative real-time polymerase chain reaction.

identified three DElncRNA-DEmiRNA interactions involving one miRNA (hsa-let-7g) and three lncRNAs (NR2F1-AS1, AC000403, and RP11-456K23). miRanda with expression correlation was used to identify DEmiRNADEmRNA pairs, and 40 DEmiRNA-DEmRNA interactions met the selection criteria. The DEmiRNA-DEmRNA interactions involved hsa-let- $7 \mathrm{~g}$ and $40 \mathrm{mRNAs}$.

\section{Construction of the ceRNA network and patbologic phenotype analysis}

To further explore BUC carcinogenesis and tumor progression, a ceRNA network was constructed based on the identified interactions of both DElncRNA-DEmiRNA and DEmiRNA-DEmRNA pairs. Hub miRNAs link the above two parts of the network. In this study, hsa-let$7 \mathrm{~g}$ was the hub element of the ceRNA network bridging the DElncRNA-DEmiRNA-DEmRNA interactions. In addition, three lncRNAs (NR2F1-AS1, AC000403, and RP11-456K23) were identified as potential regulatory elements of hsa-let-7g. RIP assays were performed to confirm physical interactions between hsa-let- $7 \mathrm{~g}$ and the above three lncRNAs. The results revealed significantly enriched hsa-let-7g, NR2F1-AS1, AC000403 and RP11$456 \mathrm{~K} 23$ in the anti-Ago2 group compared to the $\mathrm{IgG}$ control group (Figure 2).

We next checked for each element within this network, which functions in prognosis. Kaplan-Meier survival analysis was performed to obtain log-rank test $\mathrm{P}$ values for each node within the network. First, the higher expression of the hub miRNA hsa-let-7g was significantly correlated with a better prognosis, with a $\mathrm{P}$ value of 0.023 . For the interacting lncRNAs, the expression of RP11-456K23 and NR2F1-AS1 was significantly associated with worse outcomes, with $\mathrm{P}$ values of 0.0055 and 0.0047 , respectively (Figure 3). There were 40 node mRNAs (ABCC9, TPM2, HSPB2, STON1, SGCA, OLFML1, GLIPR2, KLHL13, GLIPR2, PODN, HSPA12A, DCN, HSPB7, PDLIM7, KLF9, CPEB1, HAND2, TSPAN18, ALDH1B1, MYLK, MYLK, MYLK, GATA6, TPST1, HAND2, RASSF8, SYNC, HSPB2, TSPAN18, ARHGAP20, SYNC, AKT3, ADRB3, ADRB3, PDLIM3, KATNAL1, PARVA, TLN1, DCN, and STON1) in the network, among which 14 genes (ABCC9, ADRB3, ARHGAP20, CPEB1, DCN, HAND2, HSPB2, HSPB7, KATNAL1, MYLK, PDLIM3, STON1, SYNC, TPST1) whose higher expression also indicated worse outcomes (Figure 4).

We also tested the relationship between each element and tumor stage. The expression of hsa-let$7 \mathrm{~g}$ significantly decreased as BUC developed (ANOVA 

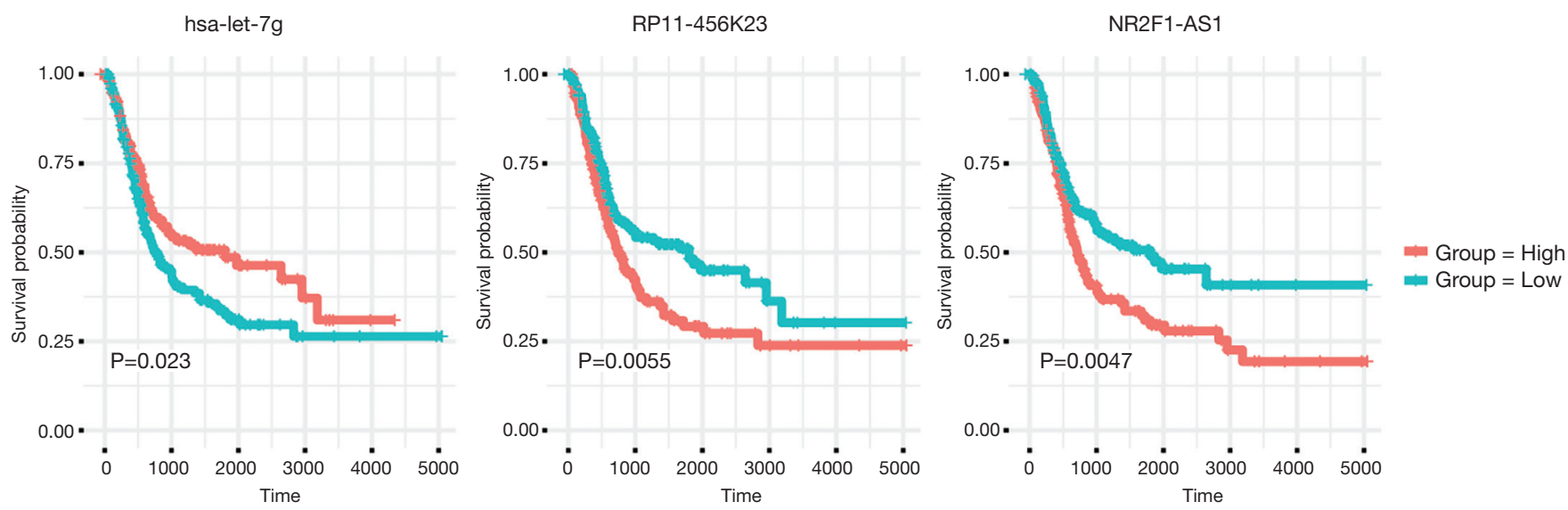

Figure 3 Kaplan-Meier survival analysis showed a significantly better prognosis for patients with higher expression of miRNA (hsa-let-7g) and a worse prognosis for patients with higher expression of lncRNAs (RP11-456K23 and NR2F1-AS1) in the ceRNA networks. lncRNA, long noncoding RNA; miRNA, microRNA; ceRNA, competing endogenous RNA.
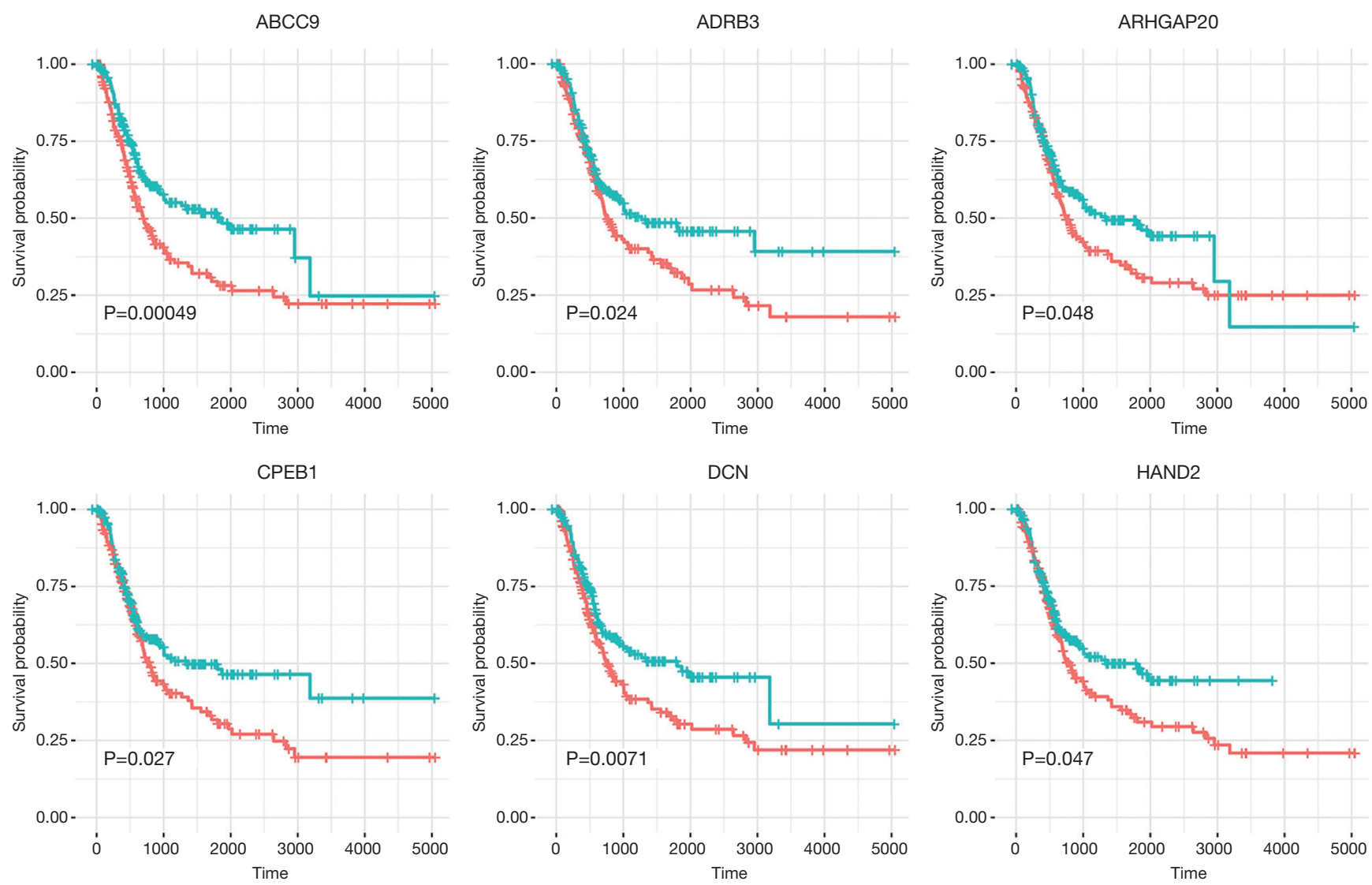

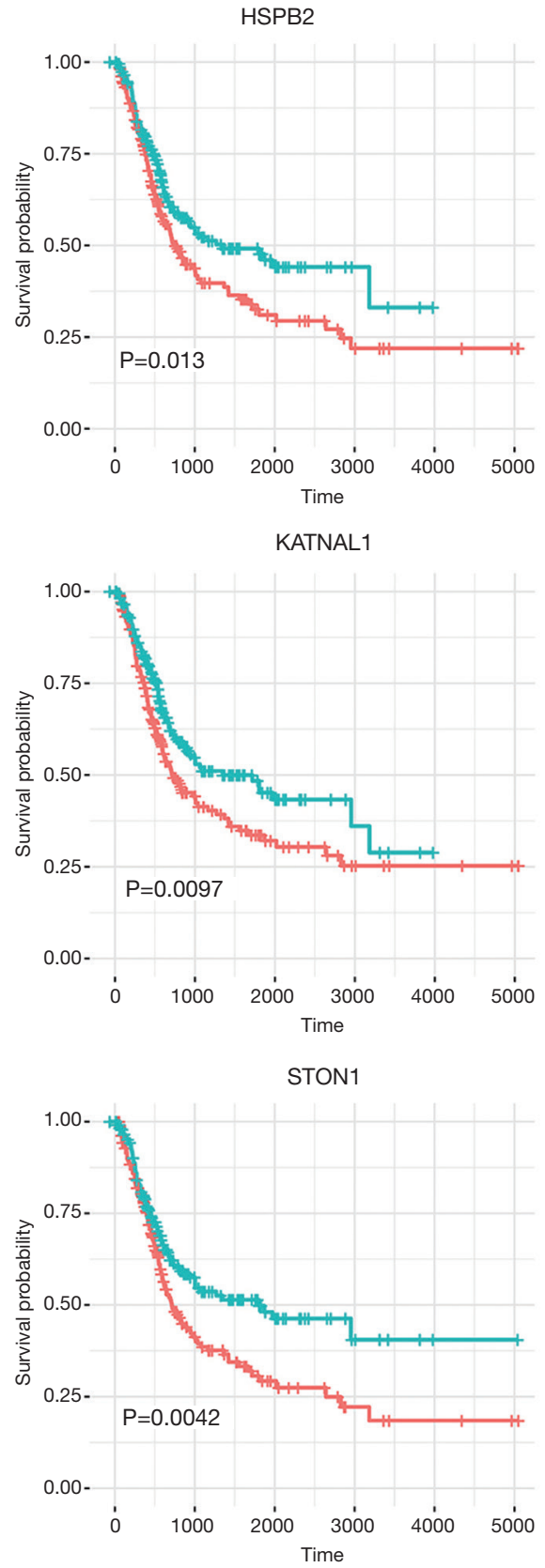

Survival

$$
\begin{aligned}
& + \text { Group }=\text { High } \\
& + \text { Group }=\text { Low }
\end{aligned}
$$

MYLK

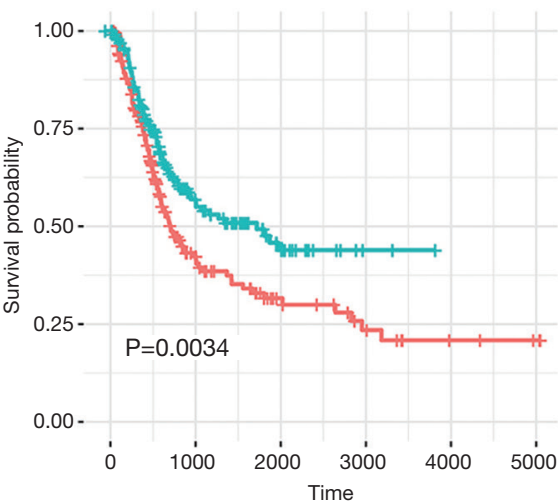

SYNC

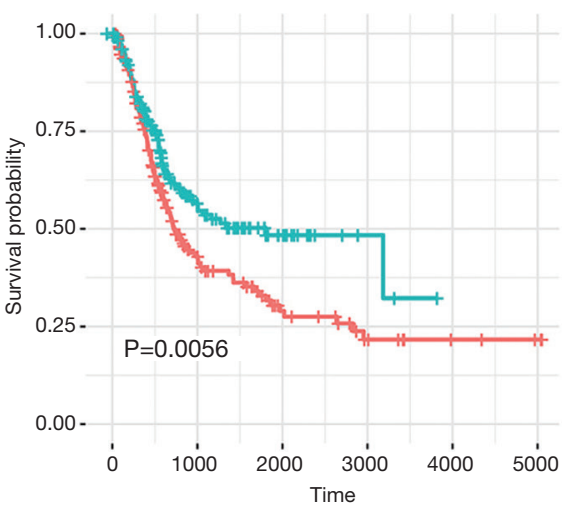

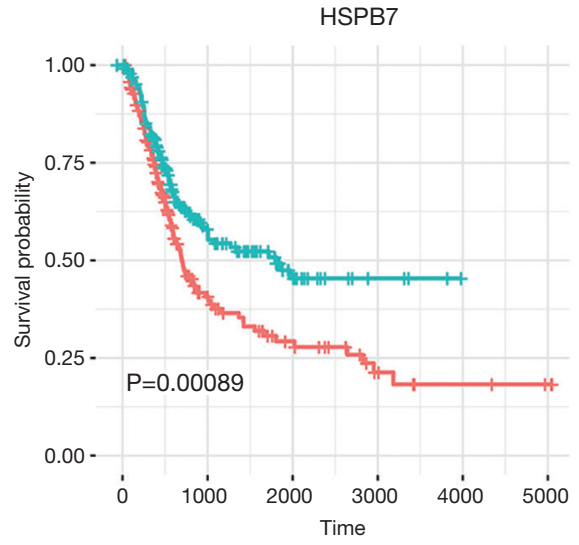

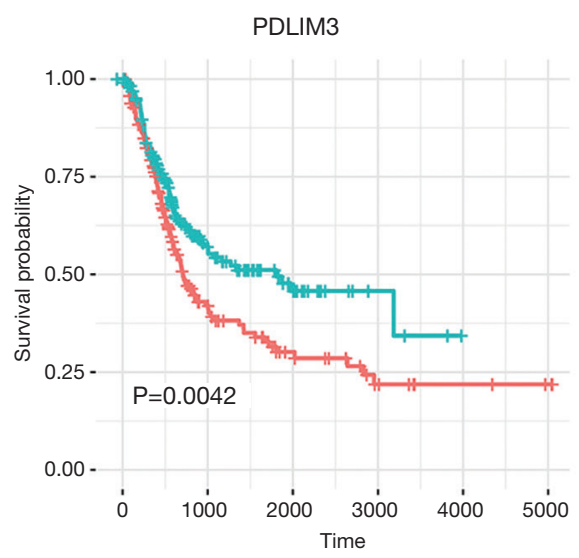

TPST1

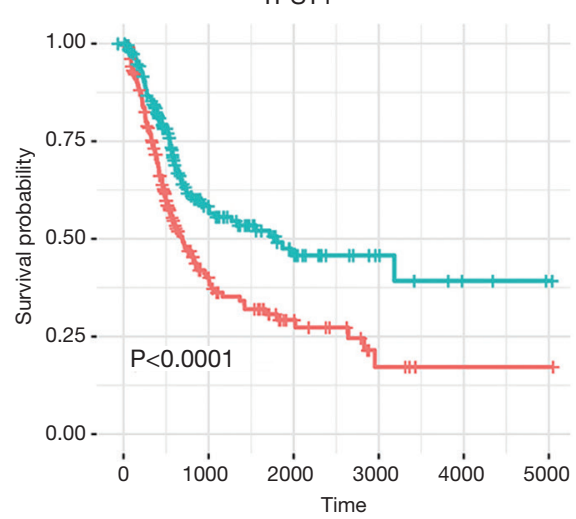

Figure 4 Kaplan-Meier survival analysis showed worse prognosis for patients with higher expression of mRNAs in the ceRNA networks. ceRNA, competing endogenous RNA.

test, $\mathrm{P}$ value $=4.4 \mathrm{e}-6)$. The upstream lncRNAs RP11456K23 and NR2F1-AS1, on the other hand, showed an obvious upward trend with $\mathrm{P}$ values of 0.0053 and 0.0015 , respectively (Figure 5). The expression of node genes predicted to be regulated by hsa-let-7g and significantly associated with prognosis was also positively correlated with tumor stage (Figure 6).

\section{Patbway enrichment analysis of the ceRNA network}

We built the final ceRNA regulatory network based on the two selected lncRNAs above, hsa-let-7g, and 14 mRNAs 

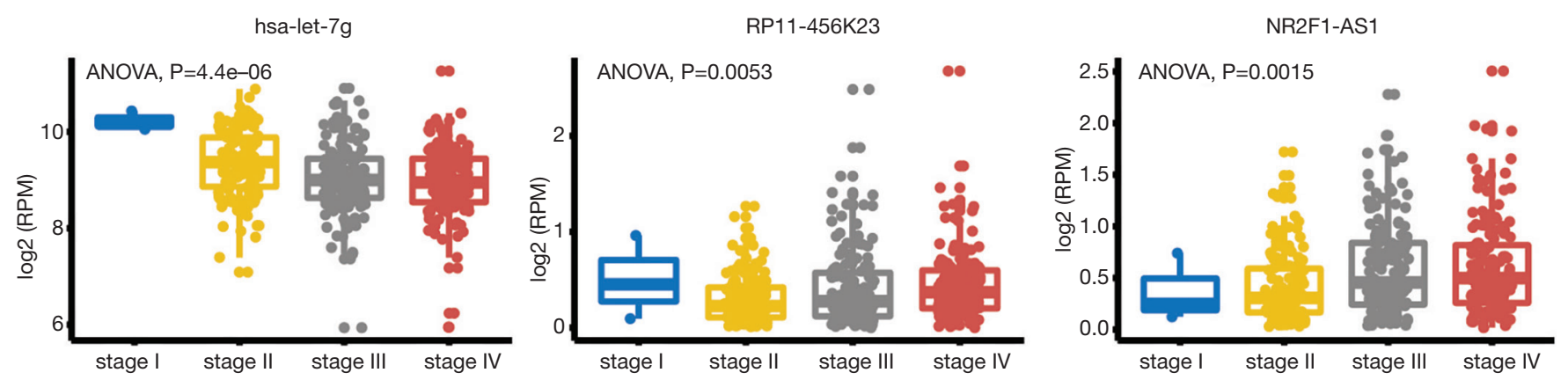

Figure 5 ANOVA showed significantly positive correlations between the expression of miRNAs (hsa-let-7g) and negative correlations between lncRNAs (RP11-456K23 and NR2F1-AS1), and tumor stages in the ceRNA networks. lncRNA, long noncoding RNA; miRNA, microRNA; ceRNA, competing endogenous RNA.

(Figure 7A). The KEGG enrichment analysis of genes in the constructed ceRNA network showed that genes were mainly enriched in the focal adhesion pathway (Figure $7 B$ ). The focal adhesion pathway is related to proteins that connect the cell skeleton to the EMC through integrins, and cancer cells exhibit highly altered focal adhesion dynamics. Tumor cell progression, migration and invasion rely on their crosstalk and the surrounding environments mediated by focal adhesion pathways. The ceRNA network we identified may mainly function in tumorigenesis, metastases and invasion through regulation of focal adhesions.

\section{Discussion}

As one of the most frequently occurring malignant tumors, it is very urgent and important to explore the molecular pathogenesis of bladder cancer. The risk of recurrence and progression for individual patients show great diversity. Stratification of patients into low-, intermediate-, and high-risk groups is essential for deciding appropriate use of adjuvant intravesical chemotherapy or bacillus Calmette-Guérin (BCG) instillations (1). More precise stratification method and other promising therapeutic targets for BUC are still rare. The ceRNA network refers to a gene expression regulation network in which lncRNAs and mRNAs crosstalk with each other through common interacting miRNAs. This ceRNA regulatory system greatly expands the functional genetic information and helps to decipher the underlying pathogenesis of human polygenic disease at the posttranscriptional level. More and more researches have shown that ceRNA interactions participate many important biological processes. Disorder of such interactions may cause diseases including cancer.
ceRNA network analysis has popped up in recently years. For example, the lncRNAs CARMN, FENDRR and ADAMTS9-AS2 were shown to regulate MEG3 in Non-Muscle Invasive Bladder Cancer through sponging important miRNAs such as miR-143-3p, miR-106a-5p and miR-34a-3p (2).

In this study, we utilized multilevel TCGA bladder urothelial carcinoma (TCGA-BLCA) expression data to identify a lncRNA-miRNA-mRNA ceRNA network and its functions in oncogenesis and tumor progression. We obtained a total of 1,380 differentially expressed lncRNAs (DElncRNAs), 203 differentially expressed miRNAs (DEmiRNAs) and 4,909 differentially expressed mRNAs (DEmRNAs). Combined with predicted interaction data and expression association profiles, we identified miRNA hsa-let-7g as the hub element of the ceRNA network. Three lncRNAs (NR2F1-AS1, AC000403 and RP11$456 \mathrm{~K} 23$ ) and 40 mRNAs (ABCC9, TPM2, HSPB2, STON1, SGCA, OLFML1, GLIPR2, KLHL13, GLIPR2, PODN, HSPA12A, DCN, HSPB7, PDLIM7, KLF9, CPEB1, HAND2, TSPAN18, ALDH1B1, MYLK, MYLK, MYLK, GATA6, TPST1, HAND2, RASSF8, SYNC, HSPB2, TSPAN18, ARHGAP20, SYNC, AKT3, ADRB3, ADRB3, PDLIM3, KATNAL1, PARVA, TLN1, DCN, and STON1) were found to competitively occupy the shared binding sequence of hsa-let-7g, thus forming a lncRNA-miRNA-mRNA ceRNA network involved in BUC tumorigenesis and progression.

To gain insight into the molecular mechanisms of the identified ceRNA network, KEGG enrichment analysis was performed on the 40 mRNAs involved. The main enriched pathways were focal adhesion and proteoglycans in cancer, which are mainly associated with extracellular matrix 

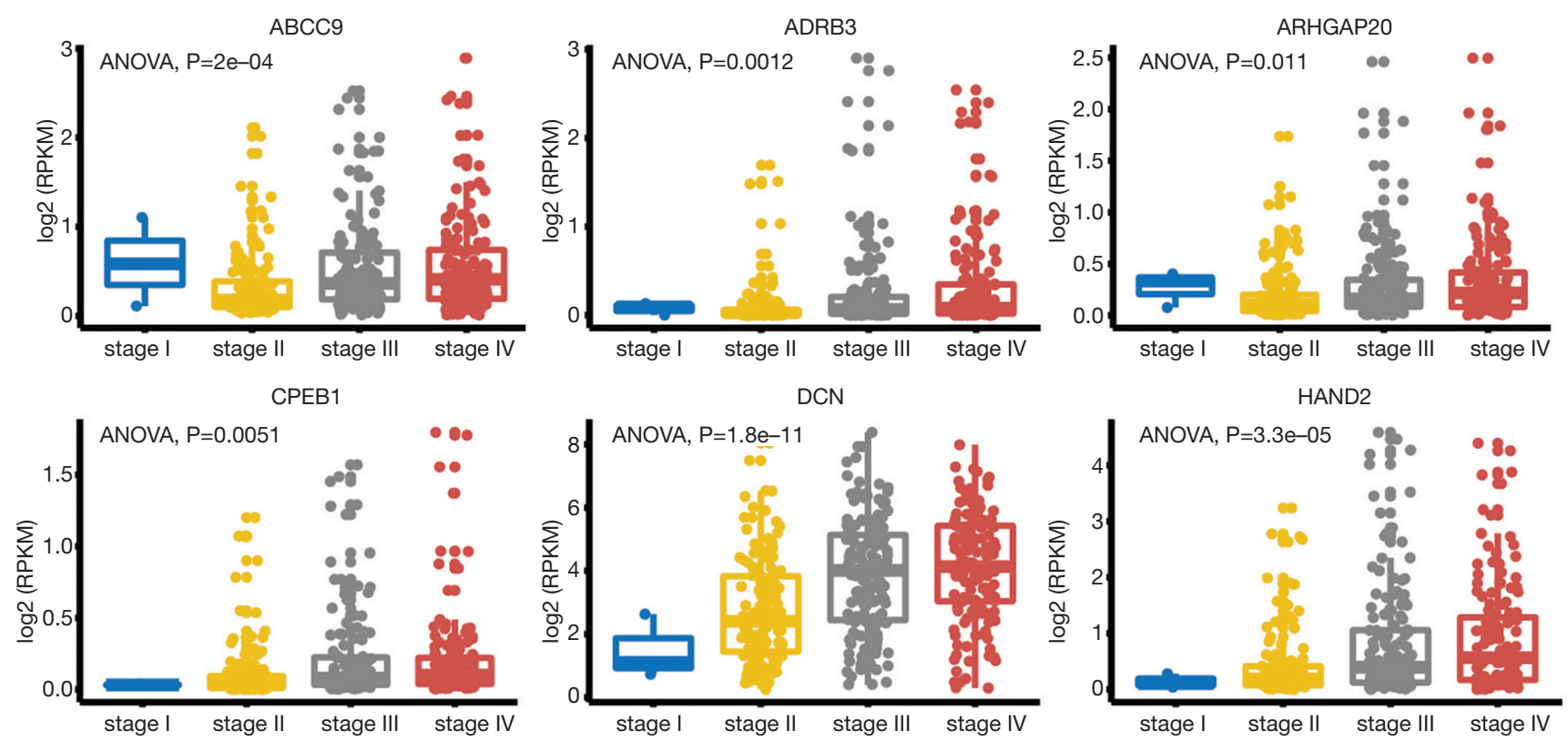

HSPB2
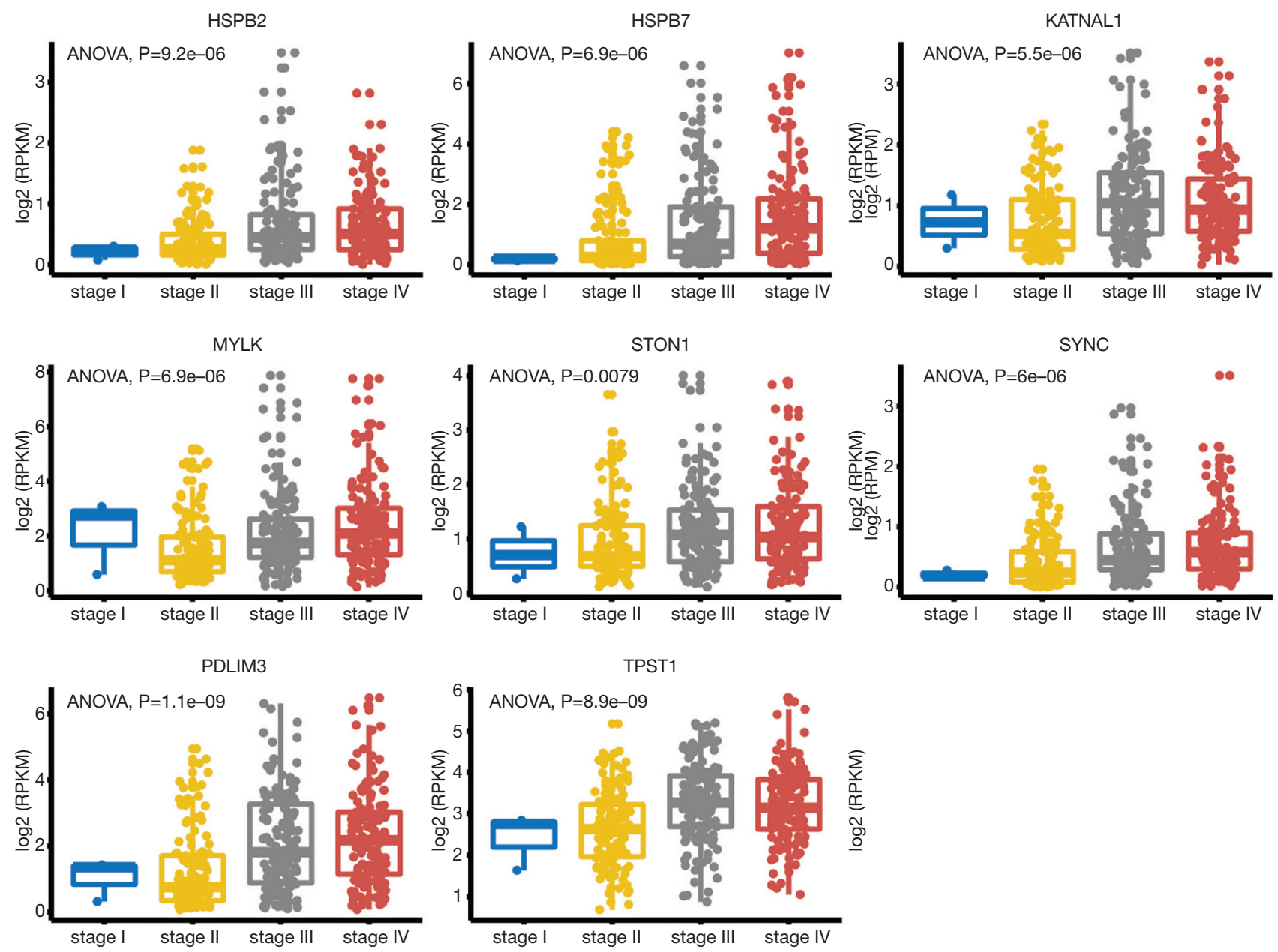

Figure 6 ANOVA showed significantly negative correlations between mRNAs and tumor stages in the ceRNA networks. ceRNA, competing endogenous RNA. 
A

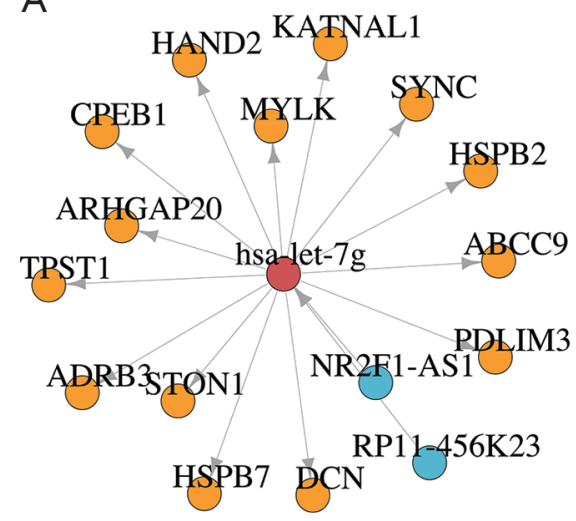

B

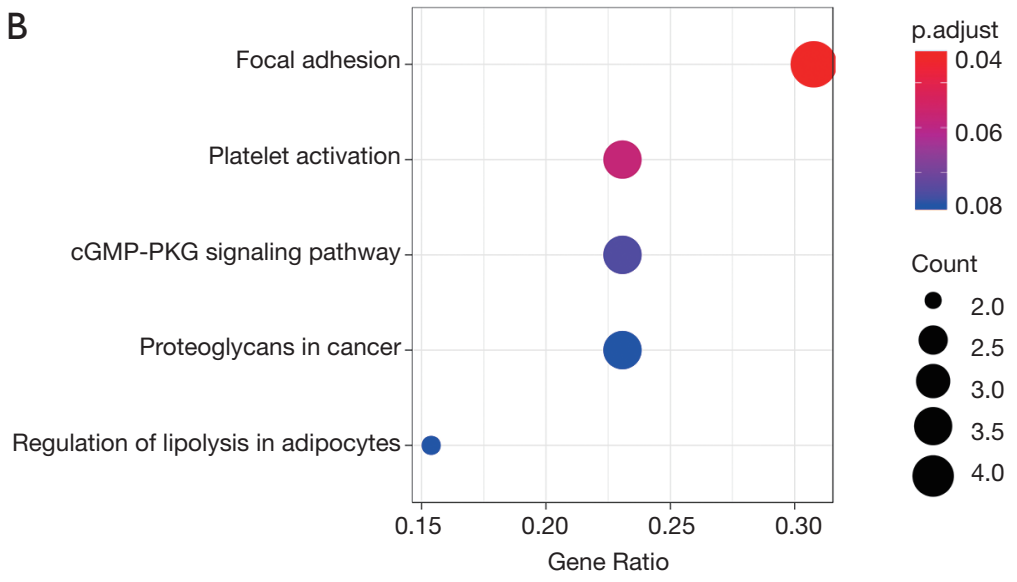

Figure 7 Pathway enrichment analysis of the ceRNA regulatory network. (A) Schematic diagram of the ceRNA regulatory network. (B) Dot plot of the KEGG pathway enrichment results. ceRNA, competing endogenous RNA.

(ECM). Focal adhesion and proteoglycans have long been reported as key influencing factors of cancer pathogenesis. The mechanisms governing the migration of cells due to regulated adhesion are crucial to the understanding of cancer cell invasion and metastasis. Targeting focal adhesion pathways has been shown to sensitize cancer cells to different treatment regimens, including radiotherapy, chemotherapy and novel molecular therapeutics (19). Proteoglycans (PGs) are important constituents of the ECM with a unique structure consisting of a protein core and glycosaminoglycan chains, which enables these molecules to act as cellular effectors important for homeostasis and contributing to disease progression (20). PGs can be classified into intracellular, pericellular, ECM proper, and intracellular with respect to their cell location. HSPB2 was identified as a novel inhibitor of apical caspase activation in the extrinsic apoptotic pathway. HSPB2 was found to be expressed in a subset of human breast cancer cell lines, and ectopic expression of HSPB2 in breast cancer cells conferred resistance to apoptosis induced by both TRAIL and TNF- $\alpha$ (21). Decorin (DCN) is the bestcharacterized member of the extracellular small leucine-rich proteoglycan family mainly associated with collagen fibrils. DCN was shown to have antifibrotic, anti-inflammatory and anticancer effects. DCN affects the biology of various types of cancers by targeting crucial signaling molecules involved in survival, growth, metastasis, and angiogenesis. Thus, DCN has also been considered a promising therapeutic molecule (22). AKT3 is one of 3 closely related serine/threonine-protein kinases (AKT1, AKT2 and AKT3) called the AKT kinase, mediating serine and/or threonine phosphorylation of a range of downstream substrates. Studies have shown that the downregulation of Akt3 significantly inhibits the growth of triple-negative breast cancer (TNBC) cell lines in 3D spheroid cultures and in mouse xenograft models (23).

LncRNAs are noncoding RNAs with lengths greater than 200 bp and play important roles in physiological and pathological processes in humans (24). One of the wellstudied functions of lncRNA is its ability to competitively bind miRNA, thus indirectly regulating mRNA. In this study, we analyzed the overall survival rate of patients associated with DElncRNAs in the ceRNA network we identified in BUC. We identified two lncRNAs associated with the prognosis of BUC. Notably, lncRNA NR2F1-AS1 was found to be upregulated in chemoresistant HCC by targeting ABCC1 via miR-363 and could promote invasion, migration and drug resistance in vitro (25).

miRNAs are one of the most well-known posttranscriptional regulatory factors, and compelling evidence has demonstrated that miRNA expression is dysregulated in human cancer. miRNAs can act as either oncogenes or tumor suppressors and have been identified as potential biomarkers for diagnosis, prognosis and therapeutic targets. Previous studies have shown that in gastric cancer (GC), hsa-let-7g can play an antitumor role under oxidative stress through the expression of DDR genes (26). In epithelial ovarian cancer (EOC), hsa-let-7g acts as a tumor suppressor and might be used to inhibit EOC tumor progression and chemoresistance to cis-platinum-based chemotherapy (27). In this study, we identified miRNA hsa-let-7g as a hub element of the ceRNA regulatory network in BUC, and higher 
expression of hsa-let-7g could predict better prognosis.

In this study, we analyzed the multilevel expression profile of BUC and identified a promising lncRNAmiRNA-mRNA regulatory ceRNA network. By integrating tumor progression and prognosis information, we ultimately determined the ceRNA network to contain two lncRNAs, one miRNA and $14 \mathrm{mRNAs}$. We validated the interactions between RP11-456K23 and NR2F1-AS1 with hsa-let-7g. The expression data from TCGA were further validated with the results of the experiments by qRT-PCR and RIP. hsa-let- $7 \mathrm{~g}$ was also suggested to regulate the expression of focal adhesion-related genes that were shown to function in metastases and invasion in other tumors. For example, the downregulation of TLN1 was shown to suppress cell proliferation, migration and invasion in nasopharyngeal carcinoma (28). MYLK can promote HCC progression by regulating the cytoskeleton to enhance epithelialmesenchymal transition (29). The elements of this ceRNA network are closely related to the molecular mechanism of BUC and serve as therapeutic targets for further treatments. Further studies are still needed to completely elucidate the detailed mechanisms of identified ceRNA network on BUC.

\section{Acknowledgments}

Funding: None.

\section{Footnote}

Reporting Checklist: The authors have completed the STROBE reporting checklist. Available at http://dx.doi. org/10.21037/tau-20-1167

Conflicts of Interest: All authors have completed the ICMJE uniform disclosure form (available at http://dx.doi. org/10.21037/tau-20-1167). The authors have no conflicts of interest to declare.

Ethical Statement: The authors are accountable for all aspects of the work in ensuring that questions related to the accuracy or integrity of any part of the work are appropriately investigated and resolved. The study was conducted in accordance with the Declaration of Helsinki (as revised in 2013). All data from TCGA are publicly available and their use do not require the approval of a local ethics committee.

Open Access Statement: This is an Open Access article distributed in accordance with the Creative Commons Attribution-NonCommercial-NoDerivs 4.0 International License (CC BY-NC-ND 4.0), which permits the noncommercial replication and distribution of the article with the strict proviso that no changes or edits are made and the original work is properly cited (including links to both the formal publication through the relevant DOI and the license). See: https://creativecommons.org/licenses/by-nc-nd/4.0/.

\section{References}

1. Siegel RL, Miller KD, Jemal A. Cancer statistics, 2019. CA Cancer J Clin 2019;69:7-34.

2. Brockdorff N. Noncoding RNA and Polycomb recruitment. RNA 2013;19:429-42.

3. Mercer TR, Mattick JS. Structure and function of long noncoding RNAs in epigenetic regulation. Nat Struct Mol Biol 2013;20:300-7.

4. Ulitsky I, Bartel DP. lincRNAs: genomics, evolution, and mechanisms. Cell 2013;154:26-46.

5. Chai J, Guo D, Ma W, et al. A feedback loop consisting of RUNX2/LncRNA-PVT1/miR-455 is involved in the progression of colorectal cancer. Am J Cancer Res 2018;8:538-50.

6. Yu X, Zhao J, He Y. Long non-coding RNA PVT1 functions as an oncogene in human colon cancer through miR-30d-5p/RUNX2 axis. J BUON 2018;23:48-54.

7. Bai N, Peng E, Qiu X, et al. circFBLIM1 act as a ceRNA to promote hepatocellular cancer progression by sponging miR-346. J Exp Clin Cancer Res 2018;37:172.

8. Yu C, Longfei L, Long W, et al. LncRNA PVT1 regulates VEGFC through inhibiting miR-128 in bladder cancer cells. J Cell Physiol 2019;234:1346-53.

9. Jiao D, Li Z, Zhu M, et al. LncRNA MALAT1 promotes tumor growth and metastasis by targeting miR-124/foxq 1 in bladder transitional cell carcinoma (BTCC). Am J Cancer Res 2018;8:748.

10. Chen JB, Zhu YW, Guo X, et al. Microarray expression profiles analysis revealed lncRNA OXCT1-AS1 promoted bladder cancer cell aggressiveness via miR-455-5p/JAK1 signaling. J Cell Physiol 2019;234:13592-601.

11. Xiong Y, Wang L, Li Y, et al. The Long Non-Coding RNA XIST Interacted with MiR-124 to Modulate Bladder Cancer Growth, Invasion and Migration by Targeting Androgen Receptor (AR). Cell Physiol Biochem 2017;43:405-18.

12. Li Y, Wan B, Liu L, et al. Circular RNA circMTO1 suppresses bladder cancer metastasis by sponging miR- 
221 and inhibiting epithelial-to-mesenchymal transition. Biochem Biophys Res Commun 2019;508:991-6.

13. Giza DE, Vasilescu C, Calin GA. MicroRNAs and ceRNAs: therapeutic implications of RNA networks. Expert Opin Biol Ther 2014;14:1285-93.

14. Law CW, Chen Y, Shi W, et al. voom: Precision weights unlock linear model analysis tools for RNA-seq read counts. Genome Biol 2014;15:R29.

15. Jeggari A, Marks DS, Larsson E. miRcode: a map of putative microRNA target sites in the long non-coding transcriptome. Bioinformatics 2012;28:2062-3.

16. Betel D, Wilson M, Gabow A, et al. The microRNA. org resource: targets and expression. Nucleic Acids Res 2008;36:D149-53.

17. Tang Z, Li C, Kang B, et al. GEPIA: a web server for cancer and normal gene expression profiling and interactive analyses. Nucleic Acids Res 2017;45:W98-102.

18. Yu G, Wang LG, Han Y, et al. clusterProfiler: an R package for comparing biological themes among gene clusters. OMICS 2012;16:284-7.

19. Eke I, Cordes N. Focal adhesion signaling and therapy resistance in cancer. Semin Cancer Biol 2015;31:65-75.

20. Iozzo RV, Schaefer L. Proteoglycan form and function: A comprehensive nomenclature of proteoglycans. Matrix Biol 2015;42:11-55.

21. Oshita SE, Chen F, Kwan T, et al. The small heat shock protein HspB2 is a novel anti-apoptotic protein that inhibits apical caspase activation in the extrinsic apoptotic pathway. Breast Cancer Res Treat 2010;124:307-15.

Cite this article as: $\mathrm{Li} \mathrm{Y,} \mathrm{Zu} \mathrm{X,} \mathrm{Hu} \mathrm{X,} \mathrm{Zhao} \mathrm{C,} \mathrm{Mo} \mathrm{M,} \mathrm{Fan} \mathrm{B.}$ Competing endogenous RNA network analysis reveals pivotal ceRNAs in bladder urothelial carcinoma. Transl Androl Urol 2021;10(2):797-808. doi: 10.21037/tau-20-1167
22. Järvinen TA, Prince S. Decorin: A Growth Factor Antagonist for Tumor Growth Inhibition. Biomed Res Int 2015;2015:654765.

23. Chin YR, Yoshida T, Marusyk A, et al. Targeting Akt3 signaling in triple-negative breast cancer. Cancer Res 2014;74:964-73.

24. Gibb EA, Brown CJ, Lam WL. The functional role of long non-coding RNA in human carcinomas. Mol Cancer 2011;10:38.

25. Huang H, Chen J, Ding CM, et al. LncRNA NR2F1-AS1 regulates hepatocellular carcinoma oxaliplatin resistance by targeting ABCC1 via miR-363. J Cell Mol Med 2018;22:3238-45.

26. $\mathrm{Hu} \mathrm{H}$, Zhao X, Jin Z, et al. Hsa-let-7g miRNA regulates the anti-tumor effects of gastric cancer cells under oxidative stress through the expression of DDR genes. J Toxicol Sci 2015;40:329-38.

27. Biamonte F, Santamaria G, Sacco A, et al. MicroRNA let$7 \mathrm{~g}$ acts as tumor suppressor and predictive biomarker for chemoresistance in human epithelial ovarian cancer. Sci Rep 2019;9:5668.

28. Wang Z, Zhu Z, Lin Z, et al. miR-429 suppresses cell proliferation, migration and invasion in nasopharyngeal carcinoma by downregulation of TLN1. Cancer Cell Int 2019;19:115.

29. Lin J, He Y, Chen L, et al. MYLK promotes hepatocellular carcinoma progression through regulating cytoskeleton to enhance epithelial-mesenchymal transition. Clin Exp Med 2018;18:523-33. 